\title{
ADRC: a strategy to deal with Adaptive Optics feedback control
}

\author{
Jose M. Gonzalez-Cava*a, Luis Fernando Rodriguez Ramos ${ }^{\mathrm{b}}$, Miguel Nuñez Cagigal ${ }^{\mathrm{b}}$, Juan-Albino \\ Mendez-Perez ${ }^{\mathrm{a}}$ \\ a Departamento de Ingeniería Informática y de Sistemas, Universidad de La Laguna (ULL). Avda \\ Astrofísico Francisco Sánchez s/n. 38206 La Laguna (Tenerife), España. \\ bInstituto de Astrofísica de Canarias (IAC), C/ Vía Láctea, s/n, 38205 La Laguna (Tenerife). España.
}

\begin{abstract}
Current advanced control strategies developed in Adaptive Optics field are based on optimal techniques in order to reject the effect of atmosphere turbulence on the phase of wavefront of the incoming light. The widely researched LQG controller relies basically on models that try to capture the temporal evolution of the atmospheric wavefront. Models based on both $\mathrm{Cn}^{2}$ spatial priors and temporal dynamics have been proposed for SCAO and MOAO systems. The attractive performance of LQG is based on having an accurate model of the wavefront distortion. However, abrupt changes in conditions that directly affect the atmosphere produce a significant decrease in the LQG performance. To deal with this problem, an Active Disturbance Rejection Control (ADRC) strategy is proposed. ADRC, is an unconventional design strategy capable of overcoming the internal dynamics of the system and the external disturbances. The key idea is to treat the unknown information mainly due to atmosphere turbulence as state variable that can be estimated by an Extended State Observer (ESO). Instead of depending on a model, the controller draws the information needed to control the system from the ESO. Consequently, the control law for the Adaptive Optics system can be designed without the mathematical expression of the disturbances. As a result, the combined effect of the non-considered internal dynamics and the external disturbances is estimated and actively rejected. This contribution is focused on adapting ADRC method to Adaptive Optics requirements.
\end{abstract}

Keywords: Adaptive Optics; ADRC; Active Disturbance Rejection Control; Control Engineering;

\section{INTRODUCTION}

Light propagating through the atmosphere encounters turbulent regions in which air temperature, density and index of refraction vary. These variations result in changes in propagation speed for different rays. Consequently, an initially plane wavefront becomes distorted ${ }^{1}$. Effects like seeing, scintillation or speckle blurs the image. When the wavefront reaches the telescope, it has developed a random phase error that varies with position. As a result, the Strehl Ratio obtained (SR) as a measure of the image quality decreases considerably. Adaptive Optics is a strategy developed in 1953 which aims to improve the image quality by sensing and correcting the phase distortion introduced by the atmosphere.

From the control engineering point of view, Adaptive Optics is a really interesting problem to deal with. It is based on the well-known feedback control strategy. A Multiple-Input Multiple Output (MIMO) controller corrects the wavefront distortion by means of a deformable mirror formed by multiple actuators. The distortion is measured through a wavefront sensor. The key problem is dealing with the blurred wavefront, which is considered the main disturbance in the control system. Different projects for large telescopes demand a proper knowledge of atmospheric turbulence to design efficient adaptive optics system to reach large Strehl Ratios ${ }^{2}$. However, a proper characterization of the turbulence is not a feasible task. Proportional-Integral controller has been widely used in most applications ${ }^{3-5}$. Recently, LQG controllers have also been applied. It relies on model that tries to capture temporal evolution of the atmospheric wavefront $t^{6}$. Models based on both $\mathrm{Cn}^{2}$ spatial prior and temporal dynamics have been proposed for SCAO and MOAO systems ${ }^{7}$. However, these models have not shown accuracy enough to improve the controller performance.

The main aim of this research is to propose a new strategy to deal with this problem. Active Disturbance Rejection Control (ADRC) is supposed to be an unconventional algorithm that is able to overcome the internal dynamics of the system as well as the external disturbances ${ }^{8}$. A state observer is capable of determining the unknown information without the need of an accurate mathematical model. In fact, ADRC has already been applied in almost all domains of control engineering reaching successful results ${ }^{9-11}$. This paper is structured in the following way. After the present section, the 
basis of the Adaptive Optics is studied. Besides, the control scheme as well as the PID and LQG algorithms are shown. Then, the ADRC strategy and its application to the Adaptive Optics field are described. Finally, the conclusions and future research are presented.

\section{WHAT IS ADAPTIVE OPTICS (AO)?}

One of the main problems that astronomers have had to deal with is the image distortion caused by the atmospheric turbulence on ground-based telescopes. The atmospheric turbulence distorts images as the light rays travel at different speeds along different paths. These variations in light rays' path modify the phase producing localized phase lags/leads ${ }^{12}$. As a result, a turbulent phase is added to the original image wavefront entering the telescope. The concept of Adaptive Optics (AO) was firstly introduced by Horace W. Babcock in $1953^{13}$. He suggested that the image distortion produced by the atmosphere could be enhanced by modifying the surface of a deformable mirror (DM). The key idea was measuring the real-time image distortion and adjusting the deformable mirror to compensate the turbulent phase. The main objective was reducing the residual phase and, consequently, reducing the image distortion.

The standard scheme of an Adaptive Optics loop is shown in Figure 1. Generally, the wavefront distortion is measured by a wavefront sensor. The correction phase generated by the deformable mirror's surface is obtained through the comparison between the turbulent phase and the desirable phase (target value). A bright reference star is required to measure the blurring caused by the atmosphere effects. Different wavefront sensors technologies have been developed. However, Shack Hartmann has been the most widely wavefront sensor used in Astrophysics applications. A Shack Hartmann sensor is formed by two main parts: a lenslet array and a position-sensing detector ${ }^{14}$. The lenslet array creates a number of separated focal spots of light on the detector ${ }^{15}$. The position of these spots is directly related to the wavefront slope across the lenslet. A CCD detector determines the current focal spot position. Then, the information is converted to wavefront slopes and it makes a wavefront reconstruction possible.

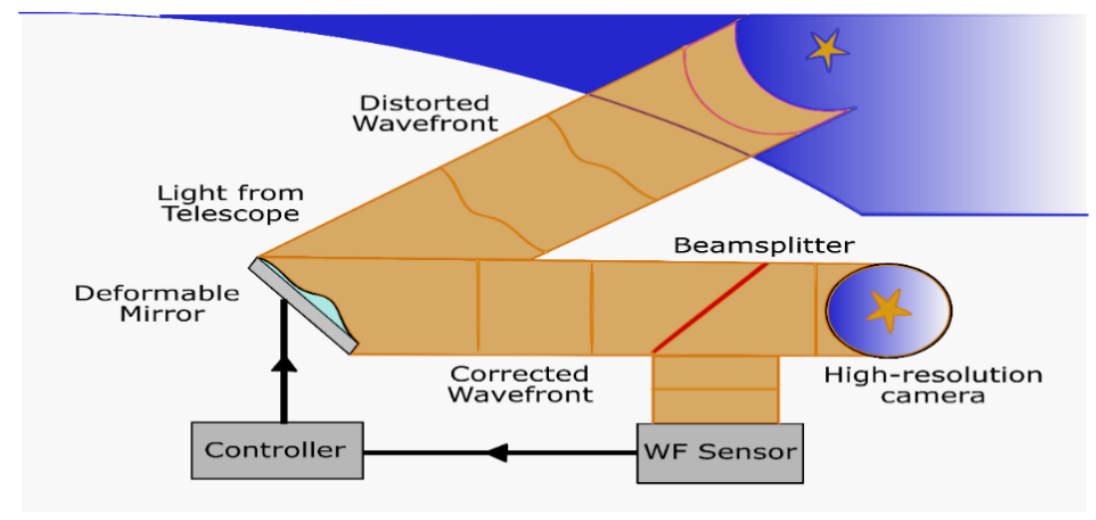

Figure 1. Standard scheme of an Adaptive Optics (AO) control loop

Many AO systems actually rely on two physically different actuator devices: a deformable mirror, whose surface can be deformed using a grid of piezo-electric or electromagnetic actuators, and a two degree of freedom tip-tilt mirror, that is usually a flat mirror able to correct tip and tilt effects ${ }^{12}$. In practice, the deformable mirror is not able to compensate all turbulence phases as the number of actuators is finite. This is known as the "fitting error". The controller is the main element capable of determining the deformable mirror appropriate signal to each actuator. The influence of an actuator displacement in the Shack-Hartmann CCD is presented in Figure 2.

\section{ADAPTIVE OPTICS FEEDBACK CONTROL}

The main aim in Adaptive Optics has been compensating the turbulent phase of the incoming light due to the atmosphere. Considering the effects of the atmosphere as an external disturbance, the traditional block diagram of the control loop developed in Adaptive Optics is shown in Figure 3. In this scheme, a zonal control is proposed. 

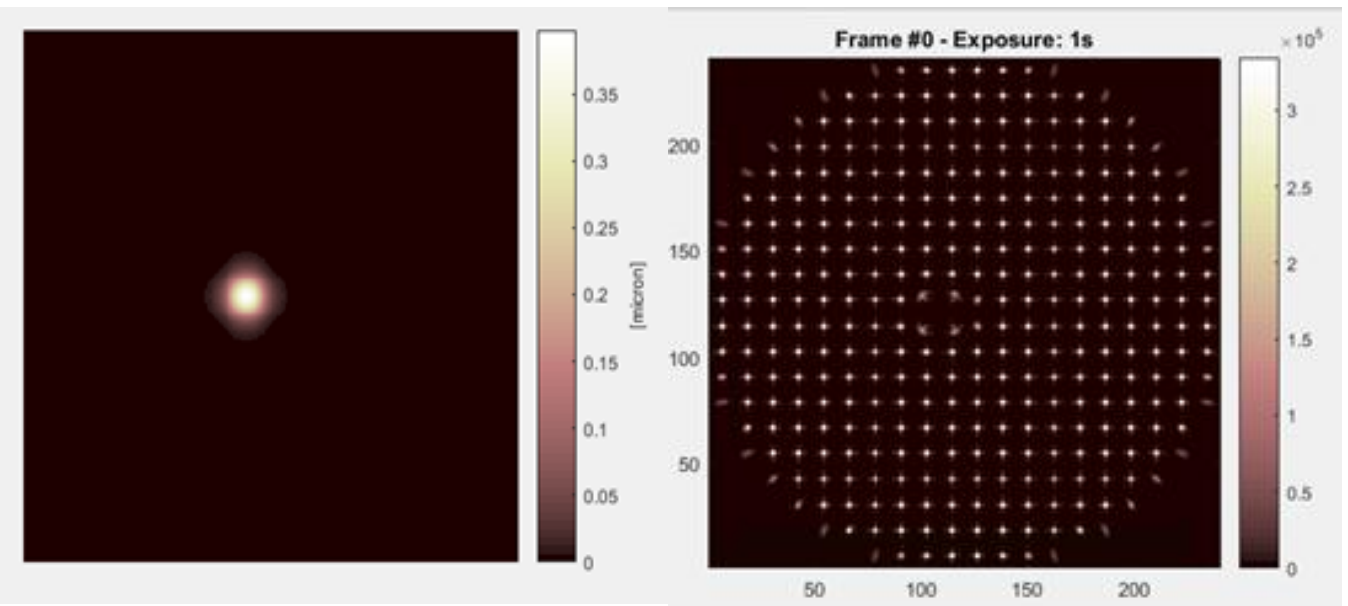

Figure 2. Spot displacement in a Shack-Hartmann sensor with 20x20 subapertures (right) due to the activation of a single Deformable Mirror actuator with $21 \times 21$ actuators (left) when a plane wavefront is received. OOMAO toolbox ${ }^{16}$.

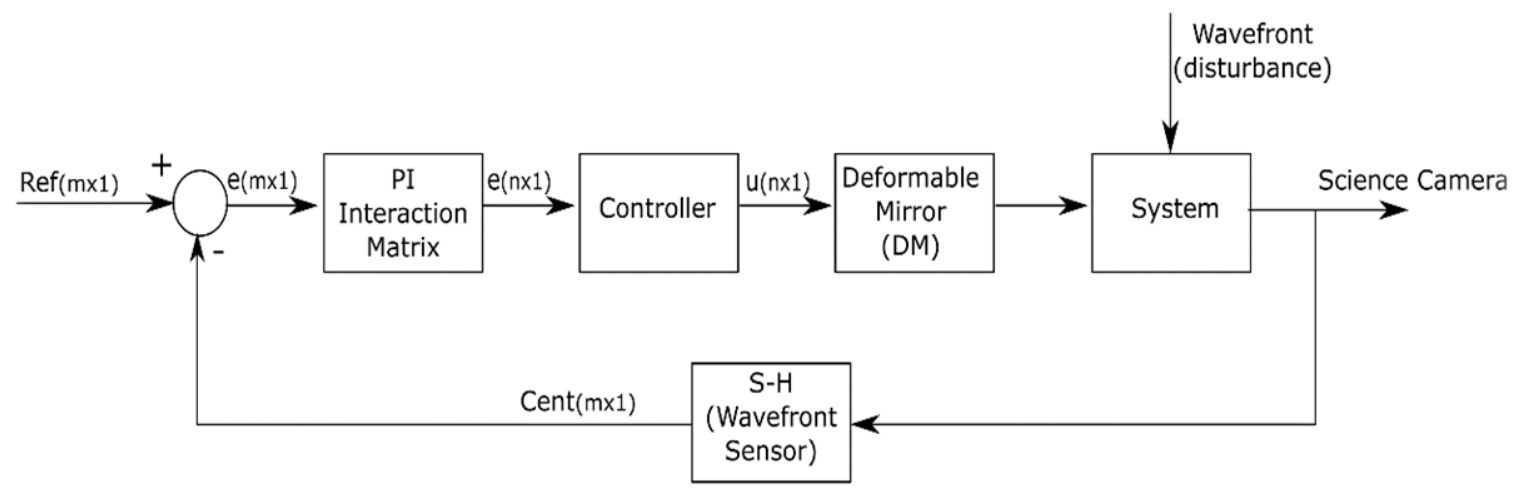

Figure 3. Block diagram of an Adaptive Optics control system.

Let $m / 2$ the number of lenslets in the sensor and $n$ the number of actuators in the DM. The WFS measures the distorted wavefront and computes the $m x 1$ centroids. The PI Interaction Matrix is the Pseudo Inverse of the rectangular Interaction Matrix that describes the linear dependences between the wavefront measurements and the corresponding actions of the deformable mirror. It is obtained through the measurement of the spots distribution when the different actuators of the deformable mirror are successively driven (poking the single actuator). The Interaction Matrix is then inverted using Single Value Decomposition with truncation. The Interaction Matrix turns the information of the sensor space (centroids) into actions in the actuator space (deformable mirror signal). As a result, a Multiple-Input Multiple-Output (MIMO) control is developed. The interaction among actuators is considered through the influence function.

Despite of existing different control strategies, only Proportional-Integral controller (PI) and, more recently, LQG algorithm have been successfully applied to the Adaptive Optics problem. In the next subsections the basis of both algorithms are explained.

\subsection{PI control}

A Proportional-Integral (PI) algorithm is a feedback control strategy widely used in most industrial control systems. The key idea is computing the controller output (electronic signal to the deformable mirror actuators) based on the error value $\mathrm{e}(\mathrm{t})$. For the Adaptics Optics field, it can be defined as:

$$
e(t)=\operatorname{ref}(t)-\operatorname{cent}(t)
$$


where $r e f(t)$ is a $m \times 1$ vector of the reference centroids for an undistorted wavefront, cent $(t)$ is a $m \times 1$ vector of the measured centroids and $e(t)$ a $m \times 1$ vector of each centroid's error. Then, the PI control structure can be defined as follows:

$$
u(t)=K_{p} e(t)+K_{i} \int e(t) d t
$$

where $K_{p}$ is the proportional gain, $K_{\mathrm{i}}$ the integral gain and $u(t)$ is a $m \times 1$ controller's output vector. $K_{i}$ can be also defined through the integral time $\left(T_{i}\right)$

$$
K_{i}=\frac{1}{T_{i}}
$$

Proportional action sets a controller output proportional to the existing error. Otherwise, the integral term increases the actuation not only considering the error but also the time for which it has persisted. The response of the system as well as the stability must be analysed in order to tune the controller parameters. An example of the application of PID controller in $\mathrm{AO}$ is shown in Figure 4.

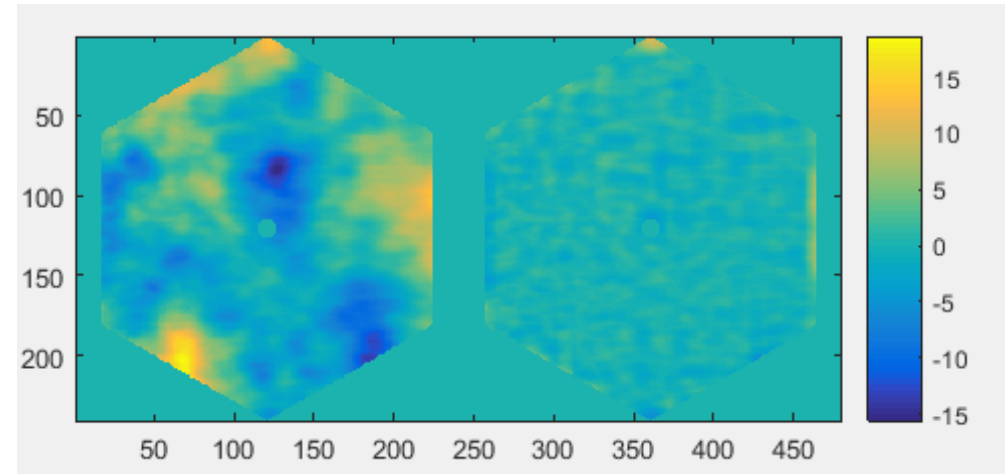

Figure 4. Original distorted wavefront phase (left) vs. corrected wavefront phase (rigth) simulated for a ten meters' telescope with a 20x20 lenslet array and 240x240 CCD wavefront resolution. 21x21 actuators' deformable mirror. PI controller $\mathrm{Kp}=1$ and $\mathrm{Ki}=0.582$. OOMAO toolbox ${ }^{16}$.

\subsection{LQG control}

In this approach the system is represented in its state space form:

$$
\begin{gathered}
\dot{x}(t)=A x(t)+B u(t)+G v(t) \\
\operatorname{cent}(t)=C x(t)+w(t)
\end{gathered}
$$

where we consider that the system has $m$ exogenous inputs, $r$ outputs, $p$ process disturbance inputs and

A = nxn system matrix

$\mathrm{B}=\mathrm{nxm}$ control input matrix

$\mathrm{C}=$ rxn observation matrix

$\mathrm{G}=\operatorname{nxp}$ system matrix

$\mathrm{x}(\mathrm{t})=\mathrm{nx} 1$ state vector

$\operatorname{cent}(t)=\operatorname{rx} 1$ output vector

$\mathrm{u}(\mathrm{t})=\mathrm{mx} 1$ control vector

$\mathrm{v}(\mathrm{t})=\mathrm{px} 1$ ramdom process noise vector

$w(t)=r x 1$ observation noise. 
The state vector is normally taken as a four-dimensional vector $(n=4)$, that includes the deformable mirror and the wavefront sensor dynamics and also the atmospheric perturbation dynamics. The output of the system is the vector of centroids.

The assumption for the disturbances variables is that $\mathrm{v}(\mathrm{t})$ and $\mathrm{w}(\mathrm{t})$ are zero-mean, uncorrelated, Gaussian, white noises, that hold

$$
\begin{gathered}
E\{v(t)\}=E\{w(t)\}=0 \\
E\left\{v(t) v^{t}(t)\right\}=V(t) \\
E\left\{w(t) w^{t}(t)\right\}=W(t)
\end{gathered}
$$

where $V(t) \geq 0$ and $W(t)>0$.

The objective is to design a feedback control law for $u$ that stabilizes the system and minimizes a cost function designed as a tradeoff between the transient response and the control effort. In particular, the cost LQG control function is

$$
J=E\left[x^{t}(T) F x(T)+\int_{0}^{T} x^{t}(t) Q(t) x(t)+u^{t}(t) R(t) u(t) d t\right]
$$

where $E[]$ denotes the expected value and $\mathrm{F}$ and $\mathrm{Q}$ are positive semidefinite and $\mathrm{R}$ is positive definite. $\mathrm{T}$ represents the final time and can be finite or infinite. The solution to the minimization problem is

$$
u(t)=-\mathrm{K}_{c} \hat{x}(\mathrm{t})
$$

where $\hat{x}(\mathrm{t})$ is an estimate of the state $x(t)$. It will be assumed that the estimator takes the form of an observer:

$$
\hat{\dot{x}}(t)=A \hat{x}(t)+B u(t)+K_{f}[y(t)-C \hat{x}(t)]
$$

$K_{f}$ is the Kalman filter gain, calculated by minimizing the error $e(T)=E\left\{e^{T}(t) e(t)\right\}$, where $e_{f}(t)=x(t)-\hat{x}(t)$, and is given by

$$
K_{f}(t)=S(t) C^{T} W^{-1}(t)
$$

where $S(t)$ is the variance of the error and can be calculated solving the Riccati equation

$$
0=A S(t)+S(t) A^{T}+G V(t) G^{T}-S(t) C^{T} W^{-1}(t) C S(t)
$$

The gain matrix $\mathrm{K}_{c}$ is:

$$
K_{f}(t)=W^{-1} B^{T} P^{-1}(t)
$$

$P$ is a symmetric positive semidefinite matrix which is the solution to the Ricatti equation:

$$
0=A^{T} P(t)+P(t) A-P(t) B R B^{T} P(t)+Q
$$

Some works have demonstrated the robustness of this strategy for the control of astronomical adaptive optics ${ }^{7,12,17}$

\section{ADRC}

The success of traditional control strategies is mostly based on an accurate knowledge about the system. As a result, the more it is known about the mathematical model of the system, the better performance is obtained when applying the control algorithms. In the feedback control strategy, widely used in most control applications, a controller manipulates the input variable and the output changes to the desirable value. Although in practice the equations that describes how the controller works are usually determined empirically, control theory establishes that the dynamics of the physical process can be captured mathematically ${ }^{18}$. Unlike the mathematical control theories, over $90 \%$ of industrial control relies on the simple proportional-integral-derivative PID type ${ }^{19}$. It is mainly due to this controller is mostly designed empirically and, therefore, a mathematical model of the process is not required. What is more, not only unknown internal 
dynamics but also external disturbances must be taken into account when facing the control problem. The question is: what do we need to know about the process in order to control it?

A proper answer was originally proposed by Han in 1998 under the name of Active Disturbance Rejection Control $(\mathrm{ADRC})^{9}$. It represents a rather drastic departure from classical as well as modern control theory. ADRC is considered as a control technology that can deal with different uncertainties including coupling of external disturbances, the system unmodeled dynamics, the zero dynamics with unknown model and the superadded unknown part of control input ${ }^{20}$. Basically, the unknown dynamics and disturbances of the plant are actively estimates and compensates by an Extended State Observer (ESO) ${ }^{21}$. Considering a general second order system without time delay described as ${ }^{22}$ :

$$
\ddot{y}+a_{1} \dot{y}+a_{0} y=b(u+w),
$$

where $u$ and $y$ are the input and output respectively. Let $w$ the external disturbance and $a_{1}, a_{0}$ and $b$ the system parameters. In this example, only the order of the system is known. The main idea is defining a $f(\cdot)$ general nonlinear, time-varying dynamic function representing the total disturbance including internal (mathematical model) and external (disturbance) uncertainties. Expression (13) can be rewritten as:

$$
\ddot{y}=b u+f,
$$

where $f=b w-a_{1} \dot{y}-a_{0} y$. Now, it is possible to consider $f(\cdot)$ as a new extended state that can be estimated in real time using a state observer.

$$
\left\{\begin{array}{c}
\dot{X}=A x+B u+E f \\
y=C X
\end{array}\right.
$$

where $A=\left(\begin{array}{lll}0 & 1 & 0 \\ 0 & 0 & 1 \\ 0 & 0 & 0\end{array}\right), B=\left(\begin{array}{l}0 \\ b \\ 0\end{array}\right), C=\left(\begin{array}{lll}1 & 0 & 0\end{array}\right)$ and $E=\left(\begin{array}{l}0 \\ 0 \\ 1\end{array}\right)$, with the state vector defined as $X=\left(\begin{array}{lll}x_{1} & x_{2} & x_{3}\end{array}\right)^{\mathrm{T}}$ $=\left(\begin{array}{lll}y & \dot{y} & f\end{array}\right)^{\mathrm{T}}$. The original system is augmented to a new third order system considering $x_{3}=f(\cdot)$. That is, $f(\dot{y}, y, w, t)$ is the generalized disturbance and the focus of this control design.

The next step is applying the observers' theory to determine the value of the total disturbance $f$. There are many observers proposed in the literature. The Extended State Observer (ESO) proposed by Han in the 1990s is an extension of the state observer in control theory ${ }^{23}$. A state observer provides an estimation of the internal state of a given real system from its input and output ${ }^{24}$. The general structure of an observer is:

$$
\dot{\hat{x}}(t)=A \hat{x}(t)+B u(t)+L(y(t)-C \hat{x}(t)),
$$

with the observer gain

$$
L=\left(\begin{array}{lll}
l_{1} & l_{2} & l_{3}
\end{array}\right)^{T},
$$

where $\dot{\hat{x}}(t)$ is the state estimation and the matrix $\mathrm{L}$ is chosen so that $\mathrm{A}-\mathrm{LC}$ is Hurwitz. The $\mathrm{n}^{\text {th }}$ order SISO nonlinear control system can be described as:

$$
x^{(n)}(t)=\mathrm{f}\left(t, x(t), \dot{x}(t), \ldots, x^{(n-1)}(t)\right)+w(t)+u(t),
$$

which can be rewritten in the canonical form:

$$
\left\{\begin{array}{c}
\dot{x} 1(t)=x 2(t) \\
\dot{x} 2(t)=x 3(t) \\
\ldots \\
\dot{x} n(t)=f(t, x 1(t), x 2(t), \ldots, x n(t))+w(t)+u(t) \\
y(t)=x 1(t)
\end{array}\right.
$$

The ESO is designed as follows:

$$
\left\{\begin{array}{c}
\dot{\hat{x}}_{1}(t)=\hat{x}_{2}(t)-\alpha_{1} g_{1}\left(\hat{x}_{1}(t)-y(t)\right) \\
\dot{\hat{x}}_{2}(t)=\hat{x}_{3}(t)-\alpha_{2} g_{2}\left(\hat{x}_{1}(t)-y(t)\right) \\
\vdots \\
\dot{\hat{x}}_{n}(t)=\hat{x}_{n+1}(t)-\alpha_{n} g_{n}\left(\hat{x}_{1}(t)-y(t)\right)+u(t) \\
\dot{\hat{x}}_{n+1}(t)=-\alpha_{n+1} g_{n+1}\left(\hat{x}_{1}(t)-y(t)\right)
\end{array}\right.
$$


where $\alpha_{i}$ are the tuning parameters, $g_{i}$ are nonlinear functions $\dot{\hat{x}}_{i}(t)$ are the approximation of the states, $\dot{\hat{x}}_{n+1}$ is the extended state $f(\cdot, t)+w(t), u(t)$ is the control input and $y(t)$ is the output (measurement). Choosing the appropriate values of $g_{i}$ and $\alpha_{1}$ make the recovery of the states as well as the extended states possible.

In addition, the first proposal introduced by Han included a Tracking Differentiator (TD) system. The main aim was using the derivative part (D) of the PID controller in presence of noise. It is well known that the derivative term can significantly improve the capability and transient performance of the control system. However, the derivative error is not easy to measure and the classical differentiation most often magnifies the noise. To overcome this difficulty, Han proposed a noise tolerant tracking differentiator:

$$
\left\{\begin{array}{c}
\dot{x} 1(t)=x 2(t) \\
\dot{x} 2(t)=r^{2} f\left(x 1(t)-v(t), \frac{x 2(t)}{r}\right),
\end{array}\right.
$$

where $r$ is the tuning parameter and $f(\cdot)$ is an appropriate nonlinear function. The effects of applying the tracking differentiator is shown in Figure 5.
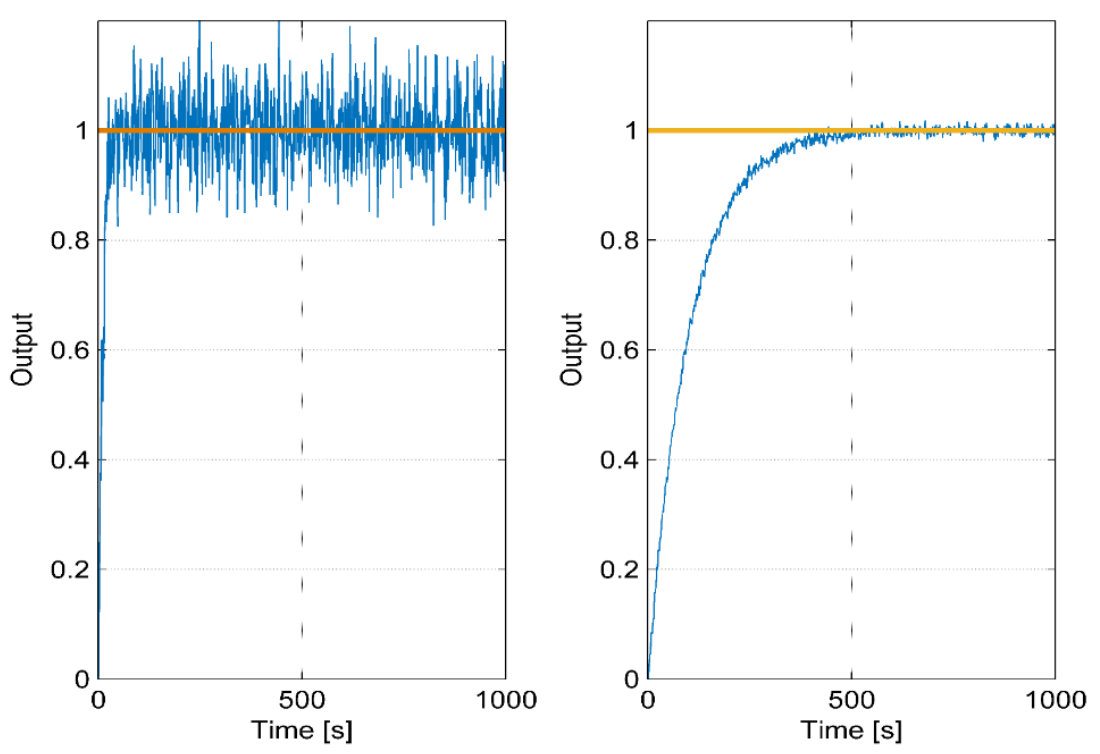

Figure 5.Response of a system affected by gaussian noise (left) and the application of the tracking differentiatior (right) proposed by Han.

All these elements introduced by Han are used to design a feedback control scheme as shown in Figure 6. The control purpose is to design an output feedback control law that drives the output of the system to track a given reference signal $\mathrm{v}(\mathrm{t})$. The first step is to design the tracking differentiator to avoid magnifying measured noise. Then, it is necessary to estimate through the ESO the system state and the "total disturbance". Finally, the feedback control compensates the total disturbance.

As a result, ADRC developed by Han has two fundamental breakthroughs: the idea of the canonical form and the use of and the concept of extended state or total disturbance ${ }^{21}$. The control of a complex nonlinear, time-varying and uncertain process is reduced to a simple problem by a direct and active estimation and rejection (cancellation) of the generalized disturbance. The main advantage is that not analytical expression of $f(y, y, w, t)$ is necessary here. Only the order of the differential equation of the system should be known. 


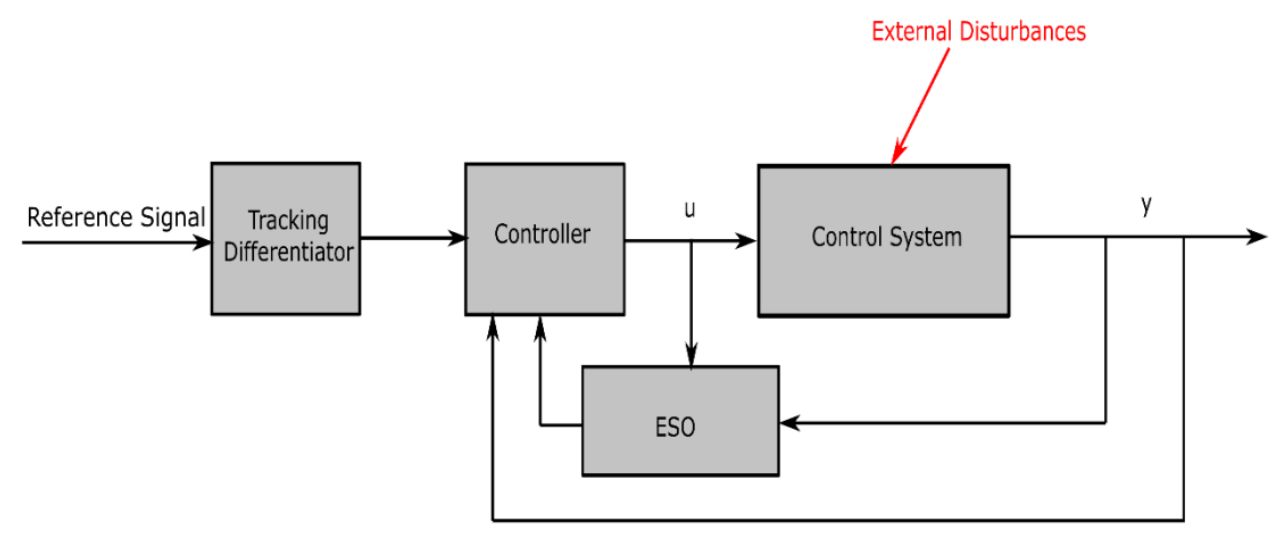

Figure 6. Topology of Aactive Disturbance Rejection Control (ADRC) proposed by Han.

\section{ADRC CONTROL IN ADAPTIVE OPTICS}

Adaptive Optics control has to deal with different uncertainties. Although traditional control strategies have led to acceptable results, more research is needed in order to improve the performance of the control loop in terms of Strehl Ratio. The main system is formed by a Subsystem 1 which turns the position of the actuators into a wavefront signal. It could be understood as if a plane wavefront reaches the deformable mirror, obtaining a new wavefront due to the current shape of the actuator. In order to model this subsystem, the Interaction Matrix could be considered. Nevertheless, the incoming wavefront (main disturbance) also affects the output of the system. An initially plane wavefront becomes distorted due to different optical path lengths because of the atmospheric turbulence. As the light propagates, effects of the turbulent regions accumulate, so by the time that the wavefront reaches the ground, it has developed a random phase error. Either measuring or trying to predict the evolution of the atmosphere is not still feasible in order to include this information in a control strategy. As a result, the output will be formed by the addition of both Subsystem 1 and the atmosphere disturbance. The main system is presented in Figure 7.

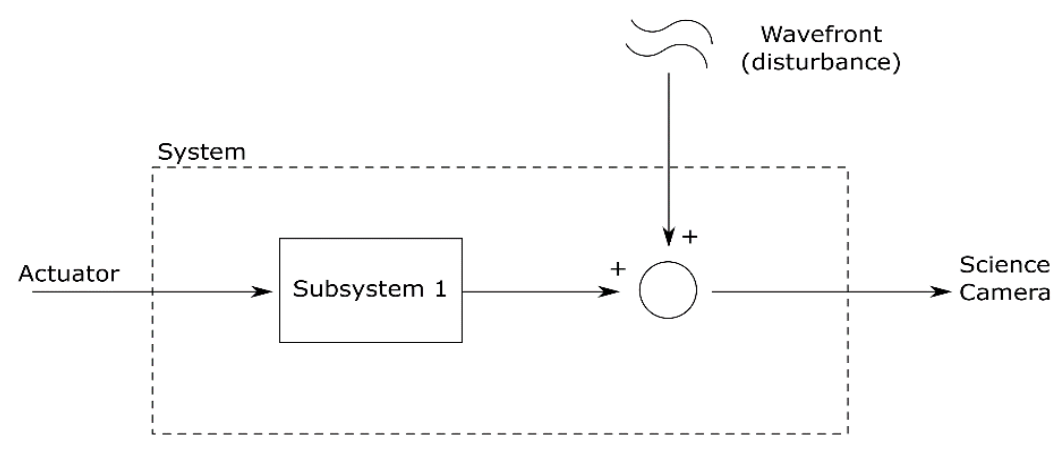

Figure 7. Scheme of the main system in the Adaptive Optics control loop.

However, not only the incoming wavefront but also another kind of disturbances can also affect the control of the system. The Shack Hartmann sensor introduces several noise sources such as readout noise or photonic noise. Consequently, it will affect the measure process as well as the calibration of the Interaction Matrix and its pseudo inverse. Time delays or sampling error must be also considered. Moreover, in order to reach a certain positions to correct the incoming wavefront, some actuators of the deformable mirror could saturate. It is another undesirable effect that is not usually taken into accoun when traditional control structures are applied.

All those facts make the Active Disturbance Rejection Control an appropriate control strategy to deal with the Adaptive Optics control. On the one hand, an initial system could be defined as the Interaction Matrix obtained through the initial 
calibration. Then, the key idea is that all the uncertainties as well as the external disturbances will be considered as an extended state or the total disturbance. A MIMO control strategy would be defined. The scheme of the proposal is shown in Figure 8. In this case, the tracking differentiator originally proposed by Han has not been included as the reference signal should keep constant (a plane wavefront).

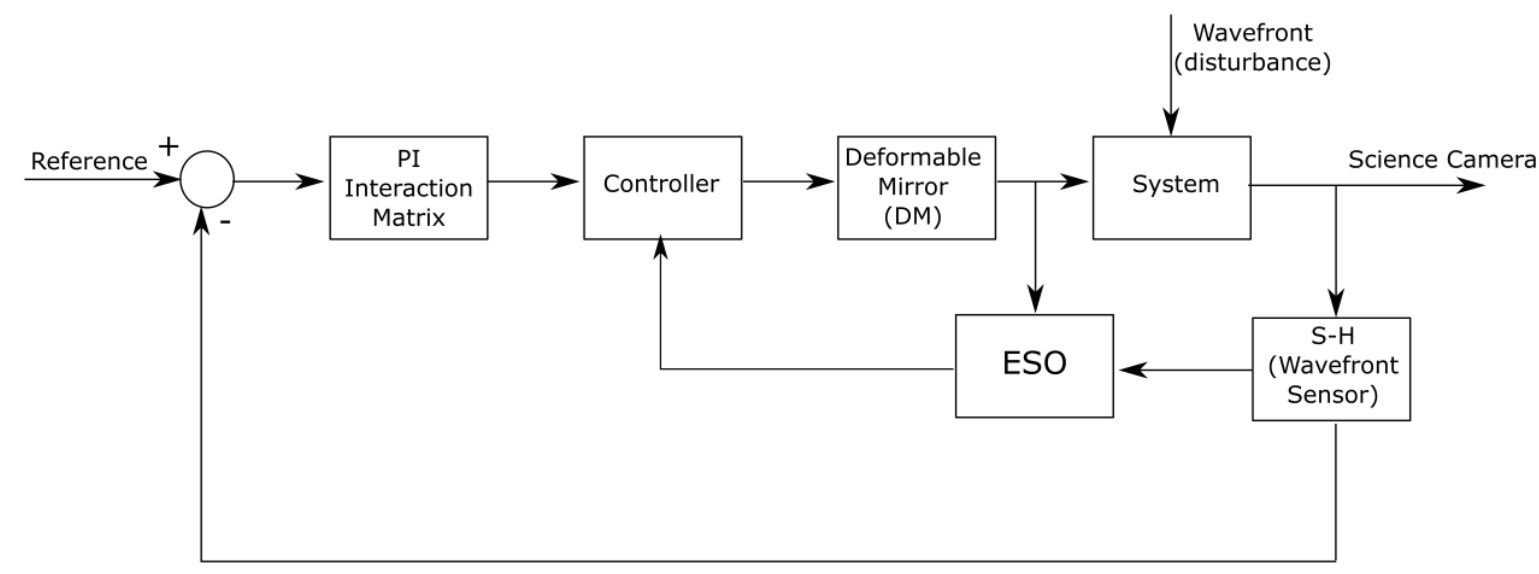

Figure 8. ADRC control block diagram for Adaptive Optics.

\section{CONCLUSIONS AND FUTURE RESEARCH}

The main objective of this paper was proposing the Active Disturbance Rejection Control as a strategy to deal with the Adaptive Optics problem. Currently, different control algorithms such as PID or LQG controllers are being applied. However, the performance of these systems is highly affected by the presence of disturbances (specifically, the input distorted wavefront) and several uncertainties when modelling dynamics. On the one hand, ADRC could afford the divergences between the proposed model and the real system. Moreover, the effects of some nonlinearity such as saturation of the actuators, dead time or even noise sources could be minimized. In conclusion, ADRC may be a strategy capable of improving the Adaptive Optics control problem without the need for an accurate mathematical model of the system or the wavefront distortion. The effect of the atmosphere turbulence can be estimated online with the Extended State Observer and being used to decouple the system from the disturbances. Furthermore, ADRC could be used not only for zonal control but also for modal control. It is a preliminary study and further research including the design of the ADRC strategy and the Extended State Observer (ESO), as well as some numerical simulations will be necessary to determine the feasibility of the algorithm in the Adaptive Optics problem.

\section{ACKNOWLEDGEMENT}

Jose M. Gonzalez-Cava's research was supported by the Spanish Ministry of Education, Culture and Sport (www.mecd.gob.es), under the 'Formación de Profesorado' grant FPU15/03347.

\section{REFERENCES}

1. BECKERS, J. M. ADAPTIVE OPTICS FOR ASTRONOMY - PRINCIPLES, PERFORMANCE, AND APPLICATIONS. Annu. Rev. Astron. Astrophys. 31, 13-62 (1993).

2. Garcia-Lorenzo, B., Eff-Darwich, A., Fuensalida, J. J. \& Castro-Almazan, J. Adaptive optics parameters connection to wind speed at the Teide Observatory. Mon. Not. R. Astron. Soc. 397, 1633-1646 (2009).

3. Wang, C. et al. Time delay compensation method for tip-tilt control in adaptive optics system. Appl. Opt. 54, 3383-3388 (2015). 
4. Zheng, Z., Li, C., Li, B. \& Zhang, S. Analysis and demonstration of PID algorithm based on arranging the transient process for adaptive optics. CHINESE Opt. Lett. 11, (2013).

5. Huang, J., Looze, D. P., Denis, N. \& Castanon, D. A. Control designs for an adaptive optics system. in PROCEEDINGS OF THE 34TH IEEE CONFERENCE ON DECISION AND CONTROL, VOLS 1-4 3753-3756 (I E E E, 1995).

6. Folcher, J.-P., Carbillet, M., Ferrari, a. \& Abelli, a. Adaptive Optics Feedback Control. EAS Publ. Ser. 59, 93130 (2013).

7. Juvénal, R., Kulcsár, C., Raynaud, H.-F. \& Conan, J.-M. LQG adaptive optics control with wind-dependent turbulent models. Proc. SPIE 9909, 99090M-99090M-7 (2016).

8. Yi, H. \& Wenchao, X. Active disturbance rejection control: Methodology and theoretical analysis. ISA Trans. 53, 963-976 (2014).

9. Qing, Z. Q. Z. \& Zhiqiang, G. Z. G. On practical applications of active disturbance rejection control. Control Conf. (CCC), 2010 29th Chinese 6095-6100 (2010). doi:10.7641/CTA.2013.31087

10. Sun, B. S. \& Gao, Z. Q. A DSP-based active disturbance rejection control design for a 1-kW H-bridge dc-dc power converter. IEEE Trans. Ind. Electron. 52, 1271-1277 (2005).

11. Xia, Y., Shi, P., Liu, G. P., Rees, D. \& Han, J. Active disturbance rejection control for uncertain multivariable systems with time-delay. IET Control THEORY Appl. 1, 75-81 (2007).

12. Kulcsár, C., Raynaud, H.-F., Petit, C. \& Conan, J.-M. Minimum variance prediction and control for adaptive optics. Automatica 48, 1939-1954 (2012).

13. Babcock, H. W. The Possibility of Compensating Astronomical Seeing. Publ. Astron. Soc. Pacific 65, 229-236 (1953).

14. Platt, B. C. \& Shack, R. History and Principles of Shack-Hartmann Wavefront Sensing. J. Refract. Surg. 17, 573-577 (2001).

15. Neal, D. R., Copland, J. \& Neal, D. a. Shack-Hartmann wavefront sensor precision and accuracy. Proc. SPIE 4779, Adv. Charact. Tech. Opt. Semicond. Data Storage Components, 148 4779, 148-160 (2002).

16. Conan, R. \& Correia, C. Object-oriented Matlab adaptive optics toolbox. in 9148, 91486C-9148-17 (2014).

17. Folcher, J.-P. \& Carbillet, M. Numerical atmospheric turbulence models and LQG control for adaptive optics system. in OPTICAL COMPLEX SYSTEMS: OCS11 (ed. Berginc, G) 8172, (SPIE-INT SOC OPTICAL ENGINEERING, 2011).

18. Gao, Z. Active disturbance rejection control: A paradigm shift in feedback control system design. Proc. Am. Control Conf. 2006, 2399-2405 (2006).

19. Levine, W. S. The Control Handbook. Control Handb. 1566 (1996). doi:www.copyright.com (http:// www.copyright.com/)

20. Han, J. From PID to active disturbance rejection control. IEEE Trans. Ind. Electron. 56, 900-906 (2009).

21. Huang, Y. \& Xue, W. Active disturbance rejection control: Methodology and theoretical analysis. ISA Trans. 53, 963-976 (2014).

22. Zheng, Q. \& Gao, Z. Predictive active disturbance rejection control for processes with time delay. ISA Trans. 53, 873-881 (2014).

23. Huang, Y. \& Han, J. Q. Analysis and design for the second order nonlinear continuous extended states observer. CHINESE Sci. Bull. 45, 1938-1944 (2000).

24. Guo, B.-Z. \& Zhao, Z.-L. Active Disturbance Rejection Control for Nonlinear Systems. (John Wiley \& Sons Singapore Pte. Ltd, 2016). doi:10.1002/9781119239932 